\title{
Kinematics and the origin of the internal structures in HL Tauri jet (HH 151)^ (Research Note)
}

\author{
T. A. Movsessian ${ }^{1}$, T. Yu. Magakian ${ }^{1}$, and A. V. Moiseev ${ }^{2}$ \\ 1 Byurakan Astrophysical Observatory, 0213 Aragatsotn reg., Armenia \\ e-mail: tigmov@bao.sci.am; tigmag@sci.am \\ 2 Special Astrophysical Observatory, 369167, N. Arkhyz, Karachaevo-Cherkesia, Russia \\ e-mail: moisav@sao.ru \\ Received 28 November 2011 / Accepted 13 March 2012
}

\section{ABSTRACT}

\begin{abstract}
Context. Knotty structures of Herbig-Haro jets are common phenomena, and knowing the origin of these structures is essential for understanding the processes of jet formation. Basically, there are two theoretical approaches: different types of instabilities in stationary flow, and velocity variations in the flow.

Aims. We investigate the structures with different radial velocities in the knots of the HL Tau jet as well as its unusual behaviour starting from $20^{\prime \prime}$ from the source. Collation of radial velocity data with proper motion measurements of emission structures in the jet of HL Tau makes it possible to understand the origin of these structures and decide on the mechanism for the formation of the knotty structures in Herbig-Haro flows.

Methods. We present observations obtained with a $6 \mathrm{~m}$ telescope (Russia) using the SCORPIO camera with scanning Fabry-Perót interferometer. Two epochs of the observations of the HL/XZ Tau region in $\mathrm{H} \alpha$ emission (2001 and 2007) allowed us to measure proper motions for high and low radial velocity structures.

Results. The structures with low and high radial velocities in the HL Tau jet show the same proper motion. The point where the HL Tau jet bents to the north (it coincides with the trailing edge of so-called knot A) is stationary, i.e. does not have any perceptible proper motion and is visible in $\mathrm{H} \alpha$ emission only.

Conclusions. We conclude that the high- and low-velocity structures in the HL Tau jet represent bow-shocks and Mach disks in the internal working surfaces of episodic outflows. The bend of the jet and the brightness increase starting some distance from the source coincides with the observed stationary deflecting shock. The increase of relative surface brightness of bow-shocks could be the result of the abrupt change of the physical conditions of the ambient medium as well as the interaction of a highly collimated flow and the side wind from XZ Tau.
\end{abstract}

Key words. stars: winds, outflows - ISM: jets and outflows - ISM: individual objects: HH 151

\section{Introduction}

The Herbig-Haro jet from HL Tau (HH 151) was first noted by Mundt \& Fried (1983) and became one of the first discovered jets from young stellar objects (YSOs). The HH flow associated with HL Tau has an exciting history and even today the origin of some its features is not clear. For example, it starts as the relatively faint and narrow jet from HL Tau with a position angle of about $51^{\circ}$ but at a distance of about $20^{\prime \prime}$ from the star it abruptly changes direction by about $14^{\circ}$ and becomes much more brighter and wider. This strange behaviour was the main reason for several authors to consider this flow as an intersection of two jets: one from HL Tau and another from the hypothetical source VLA 1 (Mundt et al. 1987). But sub-millimeter (Moriarty-Schieven et al. 2006) and various infrared observations of this region did not confirm the existence of any source in the position of the VLA 1 . Presently the HH 151 jet is considered as a single flow from HL Tau itself. In this case one should assume that the abrupt changes in its morphology and direction

* Based on observations collected with the $6 \mathrm{~m}$ telescope of the Special Astrophysical Observatory (SAO) of the Russian Academy of Sciences (RAS), operated under the financial support of the Science Department of Russia (registration number 01-43). can be produced by variations of the conditions in the surrounding medium.

On the other hand, the neighbouring pre-main-sequence (PMS) star XZ Tau is surrounded by an elliptical shell of dimensions $1.5^{\prime} \times 2^{\prime}$, revealed by the high-resolution imaging in ${ }^{13} \mathrm{CO}(1-0)$ (Welch et al. 2000). Portions of the wall of this bubble can be seen also in the optical. Besides, XZ Tau drives a wide HH outflow (Krist et al. 1999). The relation between this flow and the bubble around XZ Tau is not clear yet, but we see that HL Tau is situated at the edge of an expanding shell of gas and dust, probably driven by the quasi-spherical wind from XZ Tau.

Another interesting feature of the HL Tau jet is that its knotty structure (typical for nearly all jets) also becomes prominent only after the bending and brightening described above. The Fabry-Pérot (FP) interferometry of these knots revealed their complex kinematical structure (Movsessian et al. 2007), with low and high radial velocity components of distinctly different morphology. In addition, these knots also demonstrate perceptible proper motions, which were first studied by Mundt et al. (1990). Other data, presented in the papers of Movsessian et al. (2007) and Anglada et al. (2007), showed a certain difference between the proper motions (PM) of the knots, obtained in $\mathrm{H} \alpha$ and $[\mathrm{S}$ II] emission lines. 
There are various theoretical approaches to explain the formation of knotty structures in the jets (Reipurth \& Bally 2001). Usually either various types of instabilities in the jets - KelvinHelmholtz instabilities in hydrodynamical (Micono et al. 1998) or magneto-hydrodynamical (Ouyed et al. 2003) models, thermal instabilities (Gouveia dal Pino \& Opher 1990) or time variations in the velocity of the flow that generate internal working surfaces (Raga \& Noriega-Crespo 1998), are considered. To prefer one of these approaches, we need more refined observational data. All previous proper motions (PM) investigations of $\mathrm{HH}$ jets were performed in narrow-band images and represent the motions integrated through the full profile of the emission line. We attempted for the first time to measure the PM in the HL Tau jet for structures with different radial velocities and distinct morphologies, taking into account the possibility of FP scanning interferometry to obtain the velocity-channel images with very high spectral resolution.

Thus, in this paper we present proper motions of the structures in the HL Tau jet discussed in our previous paper (Movsessian et al. 2007), obtained from scanning FP observations of this region in 2001 and 2007. We also discuss a scenario in which the formation of tilted bow-shocks and bending of the jet could be the result of interaction of the HL Tau jet and the XZ Tau wide flow. This possibility was also proposed in the our previous paper (Movsessian et al. 2007).

\section{Observations and data reduction}

Scanning FP observations in the $\mathrm{H} \alpha$ emission line were carried out at the prime focus of the $6 \mathrm{~m}$ telescope (SAO, Russia) on 10 February 2007 (seeing was 1-1.5"). We used a Queensgate ET50 etalon, operating in the 501 st order of interference (at the $\mathrm{H} \alpha$ wavelength), placed in the collimated beam of the SCORPIO focal reducer (Afanasiev \& Moiseev 2005). The SCORPIO capabilities for FP interferometry were described by Moiseev (2002). An interference filter with $F W H M=15 \AA$ centred on the $\mathrm{H} \alpha$ line cut the desired spectral range. The detector was a $2 \mathrm{~K} \times 2 \mathrm{~K} \mathrm{CCD}$ EEV 42-40 operated with $2 \times 2$ pixel binning to reduce the readout time, so $1024 \times 1024$ pixel images were obtained in each spectral channel. The field of view of about $6^{\prime}$ was sampled with a scale $0.36^{\prime \prime}$ per pixel. The spectral resolution was estimated as $F W H M \approx 0.8 \AA$ (or $\approx 36 \mathrm{~km} \mathrm{~s}^{-1}$ ) for a range of $\Delta \lambda=13 \AA$ (or $\approx 590 \mathrm{~km} \mathrm{~s}^{-1}$ ) free from overlapping orders. The number of spectral channels was 36 and the size of a single channel was $\Delta \lambda \approx 0.36 \AA\left(\approx 16 \mathrm{~km} \mathrm{~s}^{-1}\right)$.

We reduced the interferometric data with an IDL-based software developed at SAO (Moiseev 2002). After the primary data reduction, the subtraction of night-sky lines, and wavelength calibration, the observational material consists of "data cubes" in which each point in the $1024 \times 1024$ pixel field contains a 36-channel spectrum.

Proper motions were determined by comparing the velocity channels, corresponding to $-50 \mathrm{~km} \mathrm{~s}^{-1}$ and $-150 \mathrm{~km} \mathrm{~s}^{-1}$, obtained in 2001 with the same telescope and optics but another detector (see Movsessian et al. 2007), and the new ones of 2007 (all radial velocities used in this paper are heliocentric). To increase the signal-to-noise ratio $(\mathrm{S} / \mathrm{N})$ we summed the three channels in each cube around the above mentioned central velocities. The velocity range in both cases was equal to $31.5 \mathrm{~km} \mathrm{~s}^{-1}$. Then we determined the PM for both high and low radial velocity structures.

The first step of a PM measurement from FP data is to reduce the data cubes of both epochs to a common reference system.
For the HL Tau field this procedure has certain difficulties: the reference stars are scarce, and the greater part of these stars are embedded in reflection nebulae. Indeed, we were forced to use nine reference stars, including such nebulous objects as HL Tau, XZ Tau and $\mathrm{LkH} \alpha 358$, which definitely diminished the precision of astrometry. The rms of the differences in the positions of reference stars is about $0.24^{\prime \prime}$.

The "difference squared" method, described in Hartigan et al. (2001) and Reipurth et al. (2002), was used. This algorithm is well-adapted for the measurements of extended objects and works in a selected rectangular region in the images of both epochs, finding the shift in the plane of the sky, which minimizes the sum of squared differences between the intensities in the defined region. For comparison the proper motions were also determined using the barycenters of the knots. For the HL Tau jet the systematic differences between both methods were negligible for the compact high-velocity knots, but for the low-velocity bow shocks they became significant. Therefore, only results from the difference square method were used for the proper motion estimates for the bow-shocks. We estimate the total uncertainty of the position of the correlation peak and astrometry to be about $0.3^{\prime \prime}$ for the compact knots and about $0.4^{\prime \prime}$ for the extended ones.

\section{Results}

As was already described in our previous paper (Movsessian et al. 2007), the HL Tau jet consists of the high-velocity narrow jet with knotty morphology and the low-velocity bows, which are visible only in $\mathrm{H} \alpha$ and cross the jet, being located in front of the high-velocity knots. They exhibit the greater transverse widths at the greater distances from HL Tau and are not axisymmetric, being elongated in the SE direction. Their morphology is best visible in HST images (Krist et al. 2008).

The knots in HL Tau jet were described by Mundt et al. (1987) and are labelled from A to E. The connected bow-like structures were detected for knots B, C, and D only. For knot A no visible variations of the morphology in various velocities were detected; the profile of $\mathrm{H} \alpha$ emission in this knot is wide but shows no split into two velocity components. Knot $\mathrm{E}$ is visible only in the channels corresponding to high radial velocity. Thus, we estimated the PM of low and high radial velocity structures only in the knots B, C, and D. However, for the sake of completeness we measured the PM for knot $\mathrm{E}$ in the high-velocity range and for knot A using the full line profile.

The results of our PM measurements are presented in Fig. 1, where the two images of the HL Tau jet, corresponding to low and high radial velocities, with the vectors of PM of the knots, are shown. The numerical results, again separately for the low and high radial velocity structures (except for knot A), are given in Table 1.

The most obvious and interesting result is that the structural components with low and high radial velocity both have very similar values of proper motions. We will discuss this in more detail below. It is also worth mentioning that these new data confirm the tendency of the PM vectors of low-velocity $\mathrm{H} \alpha$ features to turn increasingly north with increasing distance from HL Tau, found by Movsessian et al. (2007).

When considering the motions of the knots between two epochs their shift is obvious except for knot A. Its behaviour can be described as the progressive elongation in the direction of its motion, leaving its trailing edge nearly fixed. This is clearly seen in Fig. 2, where the images of knots A and B in the two epochs, integrated by full line profile, are presented. 


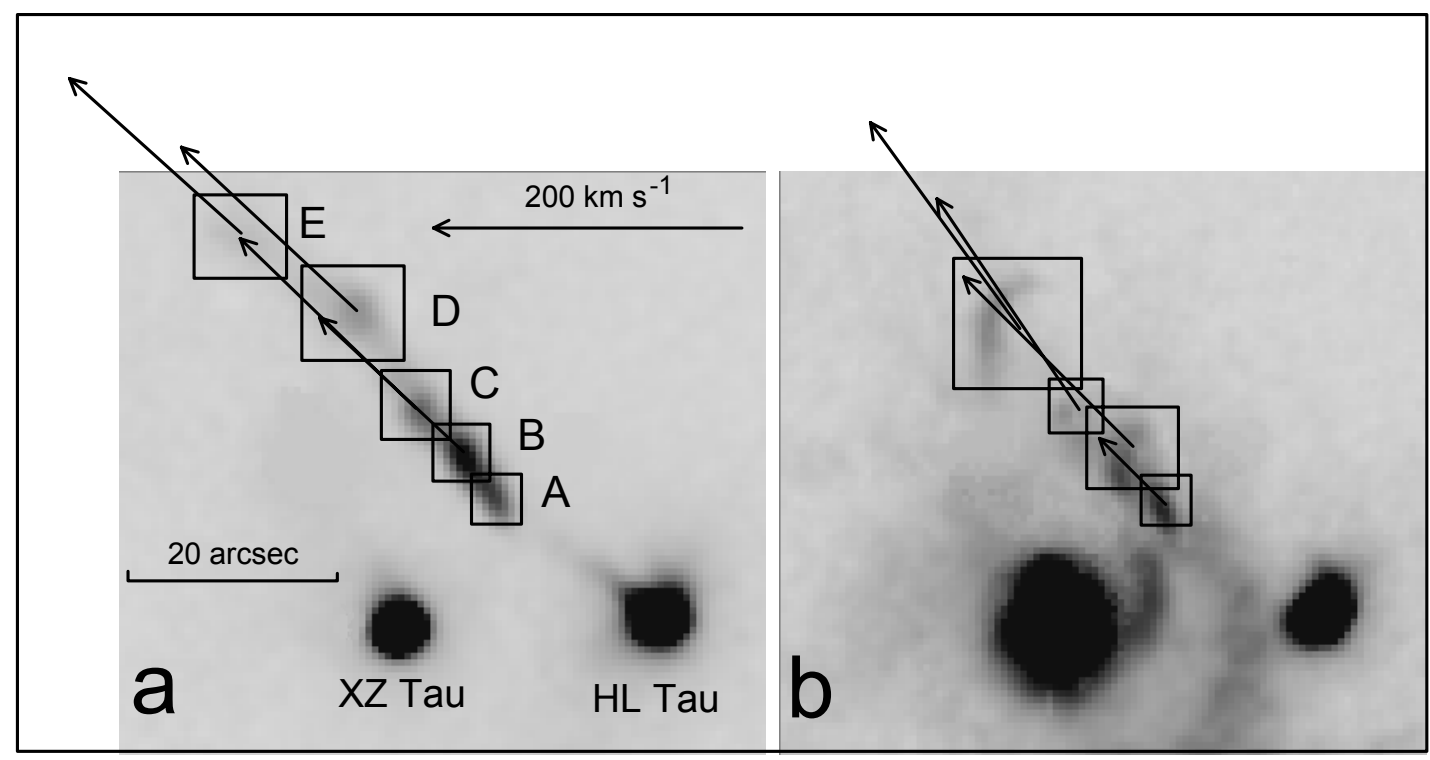

Fig. 1. Proper motions of the structures in the A, B, C, D, and E knots of HL Tau jet, corresponding to high a) and low b) radial velocities, are shown by vectors plotted in the images in $\mathrm{H} \alpha$ emission corresponding to $-150 \mathrm{~km} \mathrm{~s}^{-1}$ and $-50 \mathrm{~km} \mathrm{~s}^{-1}$, respectively. The scale of the vectors is indicated by the arrow at the top of the left panel. Rectangles show the areas used for the difference square method.

Table 1. Proper motions in the jet of HL Tau.

\begin{tabular}{lcccc}
\hline \hline Knot & $\Delta \alpha(\operatorname{arcsec})$ & $\Delta \delta(\operatorname{arcsec})$ & $V_{\mathrm{t}}\left(\mathrm{km} \mathrm{s}^{-1}\right)$ & PA $(\mathrm{deg})$ \\
\hline A & \multirow{4}{*}{13.6} & full velocity range & \\
& & 10.9 & $60 \pm 40$ & $45 \pm 19$ \\
B & 16.4 & 14.7 & & \\
C & 20.3 & 18.6 & $162 \pm 40$ & $47 \pm 12$ \\
D & 24.9 & 27.7 & $157 \pm 40$ & $46 \pm 15$ \\
E & 34.7 & 34.6 & $155 \pm 40$ & $47 \pm 19$ \\
& & low radial velocity structures & & \\
B & 17.1 & 13.7 & $154 \pm 50$ & $44 \pm 15$ \\
C & 19.8 & 17.1 & $167 \pm 50$ & $35 \pm 24$ \\
D & 27.6 & 26.6 & $161 \pm 50$ & $36 \pm 17$ \\
\hline
\end{tabular}

Notes. Column 1: Knot (nomenclature from Mundt et al. 1990); Cols. 2 and 3: right ascension and declination offsets from the position of HL Tau; Col. 4: proper motion velocity, assuming a distance of $140 \mathrm{pc}$; Col. 5: position angle.

For further clarification we show in Fig. 3 the intensity slice along the HL Tau jet in integral $\mathrm{H} \alpha$ emission. The nomenclature of the knots is the same as in Mundt et al. (1990). The shift of the knots between two epoches is apparent except at the beginning of knot A where the abrupt brightening of the flow begins. This point can be considered as stationary relative to HL Tau. The change of the relative brightness of knots A and B between 2001 and 2007 should also be noted.

Comparing the present measurements with the previous ones, we see that the directions are quite similar throughout the literature, though the values differ significantly. The present PM values are more similar to the results of Anglada et al. (2007) than to the higher estimates of Mundt et al. (1990) and Movsessian et al. (2007). Very probably this discrepancy results from the unavoidable systematic errors of various origin, which were briefly discussed also in Sect. 2. In any case, this inconsistency cannot affect the basic results and the subsequent discussion in any way.

\section{Discussion}

\subsection{Multiple working surfaces in the HL Tau jet}

The complex kinematical picture of knots in the HL Tau jet speaks about the existence of two distinct shock structures in each knot. These structures differ not only by their radial velocity but also by excitation, because bow-shape structures are visible only in $\mathrm{H} \alpha$ emission and high-velocity knots are much brighter in [S II] (Movsessian et al. 2007; Krist et al. 2008). This picture strongly resembles separation by excitation of bow-shock and Mach disk structures in the terminal working surfaces of the jets (Raga \& Noriega-Crespo 1998). This division of shocked regions was indeed found through narrow-band imagery (Reipurth $\&$ Heathcote 1992). Moreover, with the usage of spectral imagery, these regions were also separated kinematically (Hartigan et al. 2001; Morse et al. 1992; Movsessian et al. 2000). But, for the jet knots even the doubtless splitting of two shocked structures - both by radial velocity and by excitation - leaves some uncertainty in choosing between the theoretical mechanisms of the knot formation. Here, in our opinion, the proper motion values combined with the radial velocities can play the crucial role in clarifying this situation.

As we already showed, the proper motions of the two distinct radial velocity structures in each knot of the HL Tau jet have the same values. To analyse this picture we suggest considering the knots as the internal working surfaces formed in a time-dependent flow, i.e. in the same way as the terminal working surfaces. In this case they should contain the two principal 


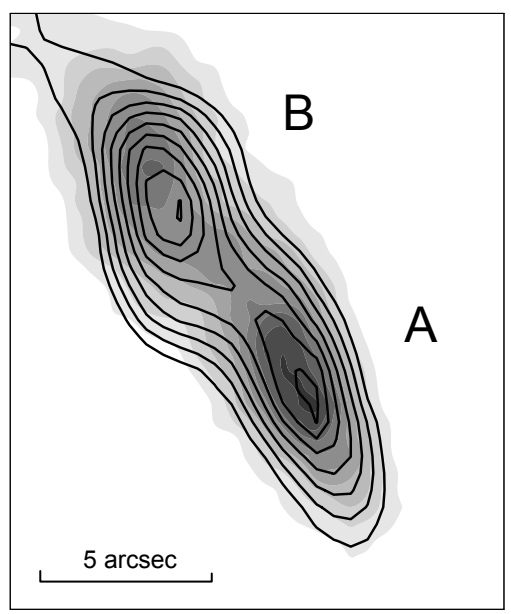

Fig. 2. Appearance of knots A and B in the HL Tau jet in 2001 (contours) and 2007 (greyscale). The progressive elongation of knot A and the obvious shift of knot B can be compared.

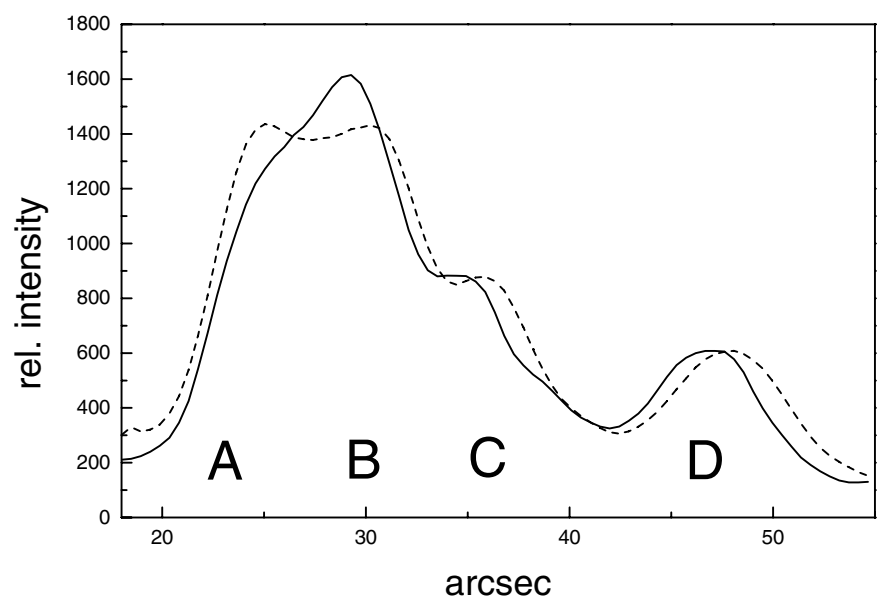

Fig. 3. Cuts along the HL Tau jet for the two epochs of observations (full line: 2001, dashed line: 2007), with the designations of the knots shown below. The intensities of both cuts are normalized by knots $\mathrm{C}$ and $\mathrm{D}$. Shifts of the knots are apparent except for the beginning of knot A.

shock regions: the "reverse shock" (or Mach disk) that decelerates the supersonic flow, and the "forward shock" (or bowshock) that accelerates the ambient medium. The pattern speed of these two structures should be the same, in contrast to the fluid speed of emitting particles.

Indeed, our data allow us to obtain for each knot both the fluid speed, which corresponds to the velocity of emitting particles, and the pattern speed of the visible structures. Tangential velocity is the projection of pattern velocity onto the plane of the sky: $V_{\mathrm{t}}=V_{\text {pattern }} \cos \alpha$, where $\alpha$ is the angle between the outflow axis and the plane of the sky. Accordingly, the radial velocity of the knot corresponds to the projection of the velocity of emitting particles (fluid speed) on the line of sight: $V_{\mathrm{r}}=V_{\text {fluid }} \sin \alpha$.

The ratio $\zeta$ of pattern speed to fluid speed was for the first time determined for HH 34 jet by Eislöffel \& Mundt (1992), but the kinematical structures in the knots were not separated in their work; only the spectral imagery technique allows this.

To obtain the correct estimates for the jet absolute velocity and inclination ( $\alpha$ angle) we need to use the structures where $\zeta=1$. For example, in a Mach disk by definition the fluid and pattern velocities have nearly the same values, so $\zeta$ should be near unity. Thus, combining the tangential and radial velocities of high-velocity compact knots (which we assume to represent Mach disks), we find that $\alpha=50^{\circ} \pm 10^{\circ}$ and the mean value of the absolute velocity of the jet is about $250 \mathrm{~km} \mathrm{~s}^{-1}$.

Previous observational estimates for $\alpha$ put its value in the range $40^{\circ}-60^{\circ}$ (Anglada et al. 2007; Movsessian et al. 2007). On the other hand, models of the HL Tau circumstellar dusty environment provide an angle of the flow about $43^{\circ}$ (Men'shchikov et al. 1999). As one can see, the new values agree well with the previous estimates.

Now we can consider the low radial velocity, bow-shape structures, which we assume to be the shock waves that entrain particles and rise the temperature of the ambient medium. Knowing the inclination angle, we obtain the mean value $\zeta=2.6$ for the three bows in the HL Tau jet, which strongly differs from the compact knots.

As was shown by Hartigan (1989), the ratio of radial velocities in shocked structures depends on the flow and ambient medium densities as well as on the jet velocity itself. From the same model, it follows that the relative surface brightness of a Mach disk and a bow-shock also depends on the ratio of the jet and ambient medium densities ( $n_{\mathrm{j}}$ and $n_{\mathrm{a}}$ correspondingly), and brighter bow-shocks are formed in denser jets. For knots within the HL Tau jet, the relative surface brightness of bow-shock and Mach disk is nearly equal, so the ratio $n_{\mathrm{a}} / n_{\mathrm{j}}$ is a little more than unity. Thus, the sudden increase of brightness of the jet and appearance of bright bow-shocks, starting from the $20^{\prime \prime}$ distance from the source, could be understood as the result of an abrupt change of the physical conditions in the ambient medium, when the flow reaches the region of lower density formed by the poorly collimated outflow from XZ Tau.

Of course, one cannot exclude the possibility that this brightening can be the result of the lower line-of-sight extinction after the deflection point. However, both in our images and in Fig. 4 from the paper of Krist et al. (2008) we can see that the narrow jet is visible from the star and almost to the deflection point; no $\mathrm{H} \alpha$ emission low-velocity structures exist in this range.

\subsection{Jet-wind interaction?}

Now we can consider the unusual behaviour of the HL Tau jet, gathering all its unusual features. Some of them were already discussed in our first paper (Movsessian et al. 2007). In summary, we have the following points:

- the bend of jet at the distance of about $20^{\prime \prime}$ from the source star;

- the abrupt increase in the brightness of the jet, starting from the same position;

- position of this point is not shifted between the two epochs of observation;

- appearance of low radial velocity structures only after the bending of the jet;

- these structures have the shape of non-axisymmetric bows with brighter SE edges;

- transverse velocity gradient is observed in these low-velocity components.

We suppose that these phenomena are mutually interrelated and, as was mentioned above, that they can result from the abrupt change in the physical condition.

Deflection of the HL Tau jet to about $12^{\circ}$ could occur from the glancing collision between the jet and the cloud material, located approximately at $20^{\prime \prime}$ distance from HL Tau (Krist et al. 2008), as in case of HH 110, where the jet collides with a dense 


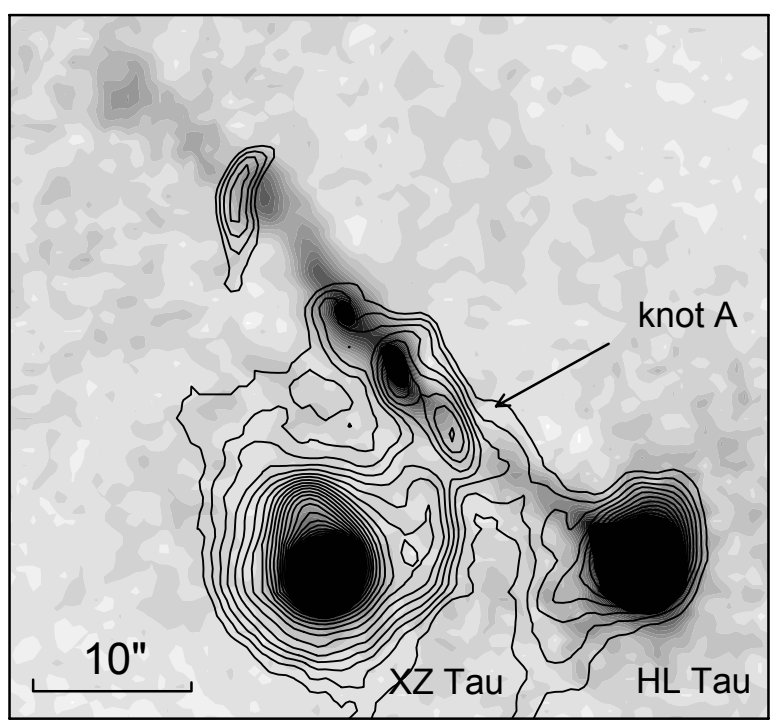

Fig. 4. Superposition of the HL/XZ Tau FP images in the monochromatic [S II] $\lambda 6716 \AA$ A line emission (greyscale) and low-velocity $\mathrm{H} \alpha$ emission (contours). The $\mathrm{H} \alpha$ observations were performed in Sept. 2001 (see Sect. 2), the [S II] data were taken in Jan. 2003 (Movsessian et al. 2007). Knot A (shown by arrow) is visible only in $\mathrm{H} \alpha$ and appears as a continuation of the $\mathrm{H} \alpha$ emission arcuate structure west of XZ Tau.

clump (Reipurth et al. 1996). But, as was mentioned above, the relative brightening of bow-shocks (which is observed in this case) takes place when the flow reaches the region with lower ambient density.

In this connection it is important to consider again knot $\mathrm{A}$. Unlike the other knots, its edge, nearest to the star, has no perceptible proper motion (Figs. 2 and 3); on the other hand, the knot is detectable only in $\mathrm{H} \alpha$ emission (Fig. 4). We are inclined to believe that the tail of knot A represents a so-called deflection shock, formed at the dense wall created by the wind from XZ Tau. Both its stationarity and morphology have a great similarity to the feature that was recently discovered in the $\mathrm{HH} \mathrm{46/47}$ jet by Hartigan et al. (2011, compare their Fig. 14 and Fig. 4 from the paper of Krist et al. 2008) and considered by them as the same kind of deflection shock.

On the other hand, the observed bending of the jet can be produced by the ram pressure of a side wind, as was shown in Ciardi et al. (2008). An expanding shell around XZ Tau, detected by Welch et al. (2000), can definitely play the role of such a side wind. As a result of this interaction, the increase of shock energy release is manifested by the brightening of the jet. The formation of elongated wings of the bows in the direction of the XZ Tau bubble (to the SE) as well as the transverse velocity gradient detected in bow-shape structures support this idea. In any case, the transverse velocity gradient is too large to be explained by jet rotation.

One cannot exclude the possibility that both mechanisms can play role in the observed behaviour of the HL Tau jet. Using the model described by Canto \& Raga (1995) we estimated, however, that the expansion velocity of XZ Tau bubble (Welch et al. 2000 ) is insufficient for the observed abrupt bend of the HL Tau jet. But, as was already mentioned above, it can be responsible for brightening of the jet.

We also recall that according to our previous measurements (Movsessian et al. 2007), confirmed also by other authors, in the SW direction from XZ Tau the definite turn in the PM vectors of the knots in the so-called "H $\alpha$ jet" is observed. As was also suggested by Movsessian et al. (2007), this can be caused by the interaction between this flow and the south-western side of XZ Tau bubble. These two cases of the unusual behaviour of the jets around the XZ Tau, in our opinion, argue that a real interaction is taking place between the jets and the expanding bubble of this star.

\section{Conclusion}

For the first time the proper motions of the knots in the collimated jet were measured with the scanning Fabry-Perót interferometer. This allows us to make certain suggestions and conclusions. In the end we summarize our findings in the following way:

(1) Our PM measurements show that the two structures distinguishable by radial velocity that exist in the HL Tau jet have the same tangential velocities, with a typical value of the order of $160 \mathrm{~km} \mathrm{~s}^{-1}$.

(2) The angle of the flow to the sky plane, determined from the radial and tangential velocities of high-velocity structures, is $50^{\circ}$ and the total space velocity of the flow is about $250 \mathrm{~km} \mathrm{~s}^{-1}$.

(3) We are inclined to assume that the different velocity structures in each knot of the HL Tau jet are detecting two principal shocks, namely bow-shock and Mach disk.

(4) Taking into account all observed features, we conclude that the knots in the jet originate from the episodic velocity variations in the HL Tau outflow, and each of them forms internal working surfaces with two principal shock regions.

(5) The strange and unusual behaviour of the HL Tau jet, starting from $\sim 20^{\prime \prime}$ from the source, is connected with the abrupt change of physical conditions in the ambient medium. Namely, the jet enters the zone where the matter is blown out by the wide wind from XZ Tau, creating a deflection shock on its border where the swept-out matter formed the dense shell.

Recent narrow-band observations of the knots in $\mathrm{HH} 34$ by Hartigan et al. (2011) demonstrated that those can also be resolved to the high- and low-excitation regions, and that in each knot both shock regions have the same proper motions. This interesting result can be considered to support our interpretation of the high and low radial velocity structures in the HL Tau jet.

We hope that this work, together with other new results, will give an impulse to develop new theoretical models of the collimated outflows, which will take into consideration all modern observational data.

Acknowledgements. The authors thank Bo Reipurth and John Bally for reading the preliminary version of the paper and for helpful discussions. Authors also express their thanks to the referee, whose comments and critical suggestions helped to improve the paper. This work was partially supported by ANSEF grant PS-astroex-2761. This work was performed partly during the stay in MPIA (T.A.M.) in Heidelberg supported by DAAD scientific exchange program.

\section{References}

Afanasiev, V. L., \& Moiseev, A. V. 2005, Astron. Lett., 31, 194 Anglada, G., Lopez, R., Estalella, R., et al. 2007, AJ, 133, 2799 Canto, J., \& Raga, A. C. 1995, MNRAS, 277, 1120

Ciardi, A., Ampleford, D. J., Lebedev, S. V., \& Stehle, C. 2008, ApJ, 678, 968 de Gouveia dal Pino, E. M., \& Opher, R. 1990, A\&A, 231, 571 Eislöffel, J., \& Mundt, R. 1992, A\&A, 263, 292 
Hartigan, P. 1989, ApJ, 339, 987

Hartigan, P., Morse, J. A., Reipurth, B., Heathcote, S., \& Bally, J. 2001, ApJ, 559, L157

Hartigan, P., Frank, A., Foster, J. M., et al. 2011, ApJ, 736, 29

Krist, J. E., Stapelfeldt, K. R., Burrows, C. J., et al. 1999, ApJ, 515, L35

Krist, J. E., Stapelfeldt, K. R., Hester, J. J., et al. 2008, AJ, 136, 1980

Men'shchikov, A. B., Henning, T., \& Fischer, O. 1999, ApJ, 519, 257

Micono, M., Massaglia, S., Bodo, G., Rossi, P., \& Ferrari, A. 1998, A\&A, 333 1001

Movsessian, T. A., Magakian, T. Yu., Amram, P., Boulesteix, J., \& Gach, J.-L. 2000, A\&A, 364, 293

Movsessian, T. A., Magakian, T. Yu., Bally, J., et al. 2007, A\&A, 470, 605

Moiseev, A. V. 2002, Bull. Spec. Astrophys. Obs., 54, 74
Moriarty-Schieven, G. H., Johnstone, D., Bally, J., \& Jenness, T. 2006, ApJ, 645,357

Morse, J. A., Hartigan, P., Cecil, G., Raymond, J. C., \& Heathcote, S. 1992, ApJ, 399, 231

Mundt, R., \& Fried, J. W. 1983, ApJ, 274, L83

Mundt, R., Brugel, E. W., \& Bührke, T. 1987, ApJ, 319, 275

Mundt, R., Ray, T. P., Bührke, T., Raga, A. C., \& Solf, J. 1990, A\&A, 232, 37

Raga, A., \& Noriega-Crespo, A. 1998, AJ, 116, 2943

Reipurth, B., \& Bally, J. 2001, ARA\&A, 39, 403

Reipurth, B., \& Heathcote, S. 1992, A\&A, 257, 693

Reipurth, B., Heathcote, S., Morse, J., Hartigan, P., \& Bally, J. 2002, AJ, 123, 362

Ouyed, R., Clarke, D. A., \& Pudritz, R. E. 2003, ApJ, 582, 292

Welch, W. M. J., Hartmann, L., Helfer, T., \& Briceno C. 2000, ApJ, 582, 292 